\title{
Tyrosine-Protein Kinase Fes/Fps
}

National Cancer Institute

\section{Source}

National Cancer Institute. Tyrosine-Protein Kinase Fes/Fps. NCI Thesaurus. Code C45498.

Encoded by human FES Gene (FES/FPS Subfamily), 822 aa 93-kDa Tyrosine-Protein Kinase FES/FPS is a tyrosine-specific protein kinase involved in normal hematopoiesis and contains an $\mathrm{FCH}$ and $\mathrm{SH} 2$ domain. 